\title{
Quantum Transport of Disordered Weyl Semimetals at the Nodal Point
}

\author{
Björn Sbierski, Gregor Pohl, Emil J. Bergholtz, and Piet W. Brouwer \\ Dahlem Center for Complex Quantum Systems and Institut für Theoretische Physik, \\ Freie Universität Berlin, D-14195 Berlin, Germany \\ (Received 26 February 2014; revised manuscript received 24 June 2014; published 11 July 2014)
}

\begin{abstract}
Weyl semimetals are paradigmatic topological gapless phases in three dimensions. We here address the effect of disorder on charge transport in Weyl semimetals. For a single Weyl node with energy at the degeneracy point and without interactions, theory predicts the existence of a critical disorder strength beyond which the density of states takes on a nonzero value. Predictions for the conductivity are divergent, however. In this work, we present a numerical study of transport properties for a disordered Weyl cone at zero energy. For weak disorder, our results are consistent with a renormalization group flow towards an attractive pseudoballistic fixed point with zero conductivity and a scale-independent conductance; for stronger disorder, diffusive behavior is reached. We identify the Fano factor as a signature that discriminates between these two regimes.
\end{abstract}

Introduction.-Topological considerations not only can be used to describe and classify band insulators and superconductors $[1,2]$, they were also found to apply to gapless phases of matter [3-10]. Perhaps the best known example of a topologically nontrivial gapless band structure is that of graphene [11], which has four topologically protected band touchings. The paradigmatic example of a topological gapless phase in three dimensions is the Weyl semimetal [12-14], which features pairs of topologically protected gap closing points in its Brillouin zone. The dispersion in the vicinity of a single isotropic nodal point can be described by the effective Hamiltonian

$$
H_{0}(\mathbf{k})= \pm \hbar v \boldsymbol{\sigma} \cdot \mathbf{k}
$$

where $v$ is the Fermi velocity, $\boldsymbol{\sigma}$ is the vector of Pauli matrices, \pm denotes the chirality, and $\mathbf{k}$ measures the Bloch wave vector relative to the momentum in the Brillouin zone at which the gap closing appears.

Weyl semimetals have attracted considerable attention due to the prediction of protected surface states with a Fermi arc [13] and the chiral anomaly in an electromagnetic response [15]. An ideal Weyl semimetal with Fermi energy at the Weyl point $\varepsilon=0$ has a vanishing conductivity $\sigma$, but a finite conductance [16], making it neither conducting nor insulating. The excitement is further fueled by the existence of concrete theoretical proposals for material candidates for Weyl semimetals, both in the solid state $[13,17,18]$ and in cold atom systems [19], as well as the experimental identification of "Dirac semimetals" [20-22], which have a pair of Weyl nodes forced to overlap by time-reversal and inversion symmetry. Although spectroscopic confirmation of a Weyl node in a real material is still lacking, magnetotransport signatures consistent with Weyl nodes were reported for BiSb [23].
An important question that concerns the comparison of theory and experimental realizations is about the stability of the Weyl nodes to the presence of disorder [24]. This question is of particular fundamental interest if the disorder is sufficiently smooth that scattering between different Weyl nodes is avoided, since disorder that does not satisfy this condition immediately removes any topological protection and leads to a trivial gapping of the spectrum and/or localization of the wave functions.

In the theoretical literature, the study of the effect of disorder on a single Weyl node, without the inclusion of electron-electron interactions, goes back to the mid 1980s $[25,26]$. Far away from the Weyl point, the expected behavior resembles that of normal metals: Disorder leads to diffusive dynamics, with a conductivity $\sigma$ that decreases with increasing disorder strength. However, unlike a normal metal, a Weyl semimetal has no transition into an Andersonlocalized phase in the limit of strong disorder [27]. Exactly at the Weyl point $\varepsilon=0$ a completely different picture emerges: There is consensus that weak disorder is irrelevant $[25,26,28,29]$, so that the vanishing density of states $\nu(\varepsilon) \propto$ $\varepsilon^{2}$ of the Hamiltonian, Eq. (1), is maintained at finite disorder strength [30,31], up to possible rare-region effects [32]. For stronger disorder, a quantum phase transition takes place, beyond which $\nu(0)$ is finite. There is no consensus for the implications of this scenario for the conductivity $\sigma$, however. Using the self-consistent Born approximation (SCBA), Ominato and Koshino [31] find $\sigma=0$ up to the critical disorder strength, and a finite conductivity that increases for stronger disorder, whereas the renormalization group approach of Ref. [29] gives a finite conductivity for subcritical disorder strengths. Boltzmann theory also gives a Weyl-point conductivity that is a decreasing function of disorder strength, but there is no critical disorder strength and $\sigma$ is finite throughout $[28,31,33]$. 
Remarkably, the question about the effect of disorder on a single Weyl node has never been put to the test numerically. Recently, similar physics has been investigated for a disordered Dirac semimetal employing diagonalization of a large tight binding model [34]. The extension of these results to a Weyl semimetal is problematic, however, because any tight binding model with a Weyl node inevitably comes with its opposite-chirality partner node [35], coupling to which cannot be fully avoided. Yet, resorting to a numerical test is particularly relevant in the present case, because none of the theoretical methods applied in the analytical theory cited above are fully controlled at the Weyl point $\varepsilon=0$ (see Ref. [29] for a critical discussion).

In this Letter, we report numerical calculations of the transport properties of a single Weyl node in the presence of a random potential. We limit ourselves to transport at the Weyl point $\varepsilon=0$, which is the energy at which the differences between a Weyl semimetal and a normal metal are most pronounced. The focus on the nodal point is not entirely academic: In contrast to the two-dimensional case (graphene or surface states of topological insulators), where unintended doping generically shifts the chemical potential away from the nodal point, in the bulk of three-dimensional Weyl semimetals $\varepsilon=0$ can be expected from the stoichiometric filling of the energy bands [30].

Our results for the conductivity are qualitatively similar to the predictions of the SCBA [31], although quantitatively the numerical results for the critical disorder strength and for the conductivity approximately differ by a factor of 2 . In the weak-disorder phase the system is better characterized by its conductance, which is finite, than by its conductivity, which is zero within the accuracy of our calculations. A transport signature that is nonzero in both phases is the Fano factor $F$, the ratio of the shot-noise power and the conductance, which we show to be an excellent indicator to discriminate between the pseudoballistic transport of the weak-disorder phase and the diffusive transport of the strong-disorder phase.

Model and numerical method.-Our numerical procedure closely follows Refs. [36,37], which considered the effect of disorder on the conductivity of graphene. We consider a Weyl semimetal of length $0<x<L$ and transverse dimensions $0<y, z<W$ with Hamiltonian

$$
H=H_{0}+U(\mathbf{r}),
$$

where $U(\mathbf{r})$ is a Gaussian random potential with zero mean $\left\langle U_{\mathbf{q}}\right\rangle=0$ and fluctuations

$$
\left\langle U_{\mathbf{q}} U_{\mathbf{q}^{\prime}}^{*}\right\rangle=\frac{K \xi \hbar^{2} v^{2}}{W^{2} L} e^{-q^{2} \xi^{2} / 2} \delta_{\mathbf{q}, \mathbf{q}^{\prime}},
$$

where $\xi$ is the correlation length and $K$ the dimensionless disorder strength. A similar random potential has been used in studies of the Dirac equation in two dimensions [36]. For $x<0$ and $x>L$ the Weyl semimetal is connected to ideal leads, which we model as Weyl semimetals with Hamiltonian $H_{0}+V$, taking the limit $V \rightarrow-\infty$ [38]. We numerically compute the transmission matrix $t$ at zero energy and determine the zero-temperature conductance using the Landauer formula $G(L, W)=\left(e^{2} / h\right) \operatorname{tr} t t^{\dagger}$ and the Fano factor $F(L)=\operatorname{tr}\left[t t^{\dagger}\left(1-t t^{\dagger}\right)\right] / \operatorname{tr} t t^{\dagger}$. To quantize transverse momenta, we apply periodic or antiperiodic boundary conditions in the $y$ and $z$ directions, and truncate at $\left|q_{y}\right|,\left|q_{z}\right| \leq 2 M / \xi$, where we verified that the results do not depend on the cutoff $M$. To ensure bulk behavior, the width $W$ is taken large enough that the results do not depend on the boundary conditions and the scaling $G \propto W^{2}, F$ independent of $W$, holds.

Pseudoballistic regime.-For the low-disorder regime, we rescale the calculated conductance $G(L, W)$ to find the dimensionless conductance $g(L)$ of a cube with linear dimension $L$,

$$
G(L, W)=\frac{e^{2} W^{2}}{h L^{2}} g(L) .
$$

In the absence of disorder, $g$ and the Fano factor $F$ are independent of $L$ [16], taking the values

$$
g_{0}=\frac{\ln 2}{2 \pi} c, \quad F_{0}=\frac{1}{3}+\frac{1}{6 \ln 2} \approx 0.574,
$$

with $c$ a numerical factor that takes the value $c=1$ (so that $g_{0} \approx 0.110$ ) for an isotropic Weyl cone. The results of numerical calculations of $g(L)$ and $F(L)$ for disorder strengths $K=1,2$, and 3 are shown in Fig. 1. The numerical data show that the presence of the random potential $U(\mathbf{r})$ leads to a bulk conductance $g$ that is always larger than the pseudoballistic value $g_{0}$, but also that the conductance $g(L)$ is a bounded function of $L$ and monotonically decreases in the large- $L$ limit. For the system sizes within our reach this decrease is most pronounced for weak disorder $(K=1)$, and less pronounced for stronger disorder $(K=3)$, which is consistent with the theoretical expectation that weak disorder is an irrelevant perturbation at $\varepsilon=0[28,29]$. The fact that $g(L)$ remains bounded as a function of $L$ is consistent with a vanishing conductivity $\sigma=0$. [A finite conductivity would correspond to $g(L) \propto L$; see the inset in Fig. 1.] The Fano factor $F$ takes the pseudoballistic value $F_{0}$ for all system sizes considered. We postpone a further discussion of these results until the end of this article.

Diffusive regime.-For stronger disorder, the conductivity $\sigma$ becomes finite. Although $\sigma$ can, in principle, be obtained from the conductance using the relation $G(L, W)=\sigma W^{2} / L$, we employ a slightly different procedure to obtain $\sigma$ from the numerically calculated conductance $G(L, W)$, in order to eliminate the effect of a finite contact resistance. Figure 2 shows the resistance 

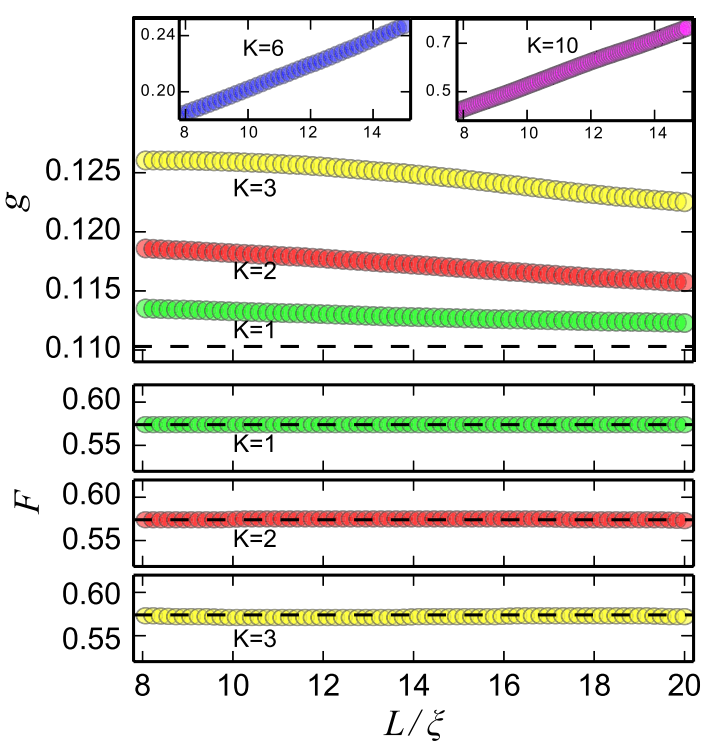

FIG. 1 (color online). Dimensionless conductance $g$ referred to a cubic sample of size $L$ (top) and Fano factor $F$ (bottom) for a single Weyl cone with a random potential for disorder strengths $K=1,2$, and 3 in the pseudoballistic regime. The data represent a disorder average over at least 10 realizations. The dashed lines refer to the clean limits $g_{0}$ and $F_{0}$ for an isotropic Weyl cone $(c=1)$. For comparison, diffusive scaling of $g$ for $K=6,10$ is shown in the insets.

$R(L, W)=1 / G(L, W)$ and the Fano factor $F(L)$ as a function of length $L$, for disorder strengths $K=6,10$, and 18. In the diffusive regime, one expects $R(L, W) \propto L / W^{2} \sigma$, so that the conductivity can be calculated as $\sigma^{-1}=W^{2} \partial R / \partial L$. We indeed observe a linear $R$ vs $L$ dependence for sufficiently large $L$. The Fano factor $F$ takes the diffusive value $F=1 / 3$ for large $L$ for the stronger disorder strengths such as $K=18$. For $K=6$ and $K=10$, the Fano factor $F$ is below the pseudoballistic limit and decreases with increasing $L$, but no limiting value could be determined for the system sizes available in our calculations. The dependence of the conductivity $\sigma$ on disorder strength $K$ is summarized in Fig. 3. We estimate that the conductivity is nonzero above a critical disorder $K_{c} \approx 5$, the behavior for $K$ just above $K_{c}$ being consistent with a linear increase $\propto K-K_{c}$ [26,31,39]. Finite-size effects prohibit a more accurate determination of the critical disorder strength. Although we adopted the expression "critical disorder strength," we note that our numerical analysis does not allow us to determine the precise nature of the transition. In passing, we also note that the conductance distribution is widest around $K_{c}$ (data not shown), a behavior well known from the three-dimensional Anderson phase transition [40].

A recent work by Ominato and Koshino [31] calculates the Weyl-point conductivity $\sigma$ using the SCBA but without further approximations, employing a correlated disorder potential compatible with the random potential used in the
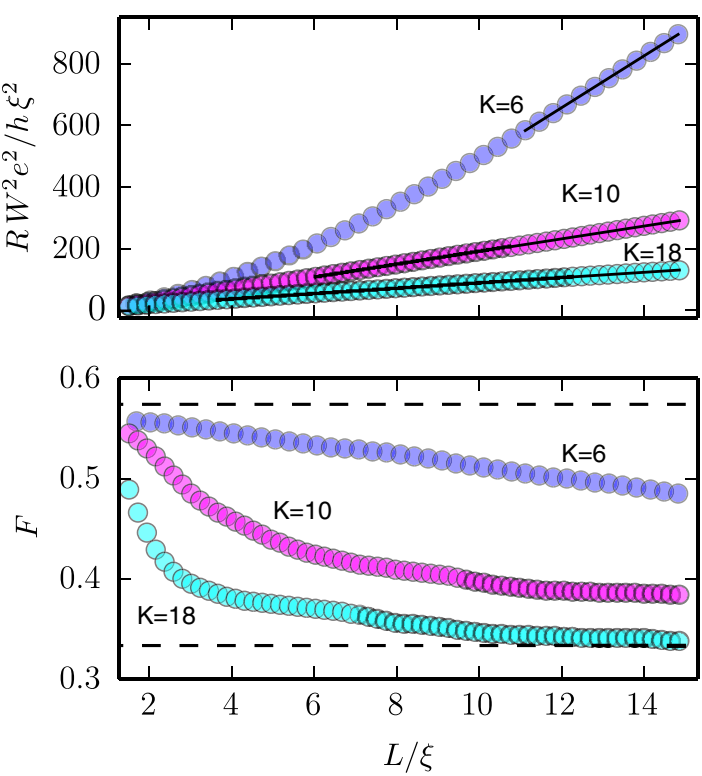

FIG. 2 (color online). Resistance $R$ (top) and Fano factor $F$ (bottom) for a single Weyl cone vs system length $L$, for disorder strengths $K=6,10$, and 18 . The thin solid lines indicate the linear fit for the conductivity $\sigma$. The dashed lines refer to the pseudoballistic and diffusive limits for the Fano factor $F$. The data represent a disorder average over at least 100 realizations.

present numerical simulation. Relating the impurity model of Ref. [31] to our Gaussian model we find a theoretical value $K_{c}^{\mathrm{SCBA}} \approx 11.3$ and a conductivity as shown by the dashed line Fig. 3 [41]. Both the value of $K_{c}^{\mathrm{SCBA}}$ and the slope of the SCBA conductivity vs disorder strength $K$ are roughly off by a factor of 2 from the numerical results.

In order to understand the quantitative failure of the SCBA, we have analyzed the corrections to the SCBA result for the self-energy $\Sigma(\mathbf{k}, \omega)$, which is related to the single-particle Green function $\mathcal{G}(\mathbf{k}, \omega)$ through the standard relation $\mathcal{G}(\mathbf{k}, \omega)=\left[\omega-H_{0}-\Sigma(\mathbf{k}, \omega)\right]^{-1}$. The diagrammatic expression for $\Sigma(\mathbf{k}, \omega)$ in the SCBA is shown in Fig. 4(a), where the double lines denote the single-particle

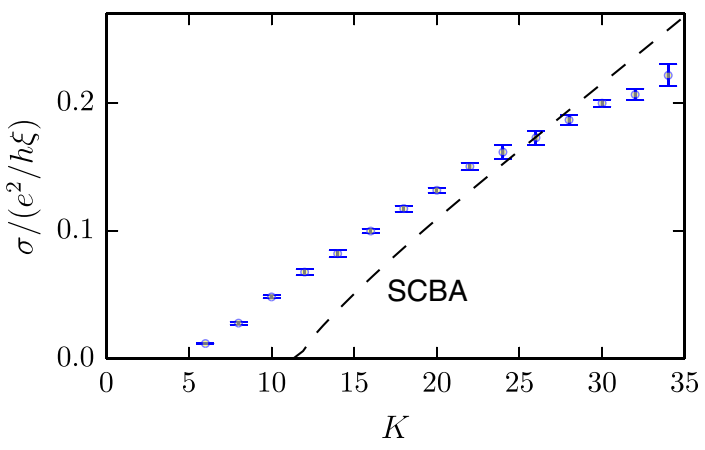

FIG. 3 (color online). Conductivity $\sigma$ for the disordered Weyl cone as a function of the disorder strength $K$. The data represent a disorder average over at least 50 disorder realizations. The dashed line refers to the SCBA theory of Ref. [31]. 


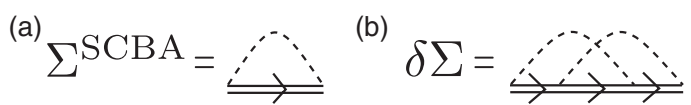

FIG. 4. Diagrammatic representation of the SCBA self-energy $\Sigma^{\mathrm{SCBA}}$ (a) and the leading correction $\delta \Sigma(\mathrm{b})$. The double solid lines denote the SCBA propagator; dashed lines are disorder correlators.

Green function $\mathcal{G}$ with $\Sigma$ replaced by $\Sigma^{\mathrm{SCBA}}$. Figure $4(\mathrm{~b})$ contains the leading correction $\delta \Sigma$ to $\Sigma^{\mathrm{SCBA}}$. The consistency of the SCBA requires that $\delta \Sigma$ is parametrically smaller than $\Sigma^{\mathrm{SCBA}}$. Indeed, for a standard disordered metal one finds $\delta \Sigma / \Sigma^{\mathrm{SCBA}}=\mathcal{O}\left(1 / k_{F} l\right)$ [42], where $k_{F}$ is the Fermi wave vector and $l$ the mean free path.

For the Weyl semimetal at zero energy one has $k_{F}=0$ and this standard argument does not apply. We have calculated the leading correction $\delta \Sigma$ at $k=0$ and $\omega=0$ using a simplified model for the disorder potential [31], in which the Gaussian correlator (3) is replaced by a cutoff at $q=2 / \xi$,

$$
\left\langle U_{\mathbf{q}} U_{\mathbf{q}^{\prime}}^{*}\right\rangle=\frac{K^{\prime} \xi \hbar^{2} v^{2}}{W^{2} L} \Theta(2 / \xi-q) \delta_{\mathbf{q}, \mathbf{q}^{\prime}}
$$

In this simplified model, one has the critical disorder strength $K_{c}{ }^{\prime}=\pi^{2}$ and the SCBA self-energy $\Sigma(0,0)^{\mathrm{SCBA}}=$ $(4 \pi i \hbar v / \xi)\left(1 / K^{\prime}-1 / K_{c}{ }^{\prime}\right) \Theta\left(K^{\prime}-K_{c}{ }^{\prime}\right)$ [31]. The calculation of the diagram of Fig. 4(b) for $K^{\prime}$ close to the critical disorder strength $K_{c}{ }^{\prime}$ then gives [43]

$$
\frac{\delta \Sigma(0,0)}{\Sigma(0,0)^{\mathrm{SCBA}}} \simeq 0.62+11\left(\frac{1}{K_{c}{ }^{\prime}}-\frac{1}{K^{\prime}}\right)
$$

which is not parametrically small. Since the simplified model, Eq. (6), does not qualitatively differ from the Gaussian model used in the numerical calculations [31], we expect that this result carries over to that case, too.

Discussion.-In the framework of Drude transport theory for normal metals, the quasiparticles at the Fermi energy are endowed with a mean free path, which becomes shorter if the disorder becomes stronger. At the same time, the presence of a random impurity potential has a negligible effect on the density of states. The result is a conductivity that decreases upon increasing the disorder strength. In contrast, for a Weyl node at the degeneracy point it is the disorder which generates the density of states [25,26,28-31], a finite density of states appearing only above a certain critical disorder strength. As a result of this vastly different physical mechanism, a Weyl node at the degeneracy point shows behavior opposite to that of a normal metal: Increasing disorder beyond the critical disorder strength leads to an increase of the conductivity. This remarkable theoretical prediction has been confirmed in our numerical calculations.
The increase in conductivity with disorder is reminiscent of the two-dimensional Dirac Hamiltonian $H_{0}^{2 d} \propto$ $v\left(k_{x} \sigma_{x}+k_{y} \sigma_{y}\right)$, for which the conductivity $\sigma$ was also found to be an increasing function of disorder strength [36,37,44]. A fundamental difference with $H_{0}^{2 d}$ is, however, that $H_{0}^{2 d}$ has a finite conductivity for all disorder strengths, whereas the Weyl semimetal at the degeneracy point requires a minimum disorder strength for diffusive behavior to set in.

For the two-dimensional Dirac Hamiltonian, the inverted dependence of conductivity on disorder strength was found to be related to the fact that $H_{0}^{2 d}$ (with a disorder term but without the condition that the disorder be smooth, because of the absence of other Dirac nodes) is the surface theory of a three-dimensional time-reversal invariant topological insulator [27]. Similarly, the surface theory of a hypothetical four-dimensional topological insulator is described by the Hamiltonian $H_{0}$ of Eq. (1). Thus, it is expected on general grounds that $H_{0}$ evades localization [27]. Our numerical results are consistent with this expectation. Indeed, although the conductivity $\sigma$ vanishes in the weak-disorder regime, the conductance $g$ remains finite. It is a finite conductance, not a finite conductivity, which is the proper signature of the absence of localization [45].

There is a subtle but important difference between the numerical calculations we performed here and the analytical theories of the conductivity cited in the Introduction: In our calculations, the conductivity $\sigma$ is obtained from the conductance $G$ of a finite-size sample, for which the energy $\varepsilon$ is set to zero at the beginning of the calculation. In contrast, in the renormalization group, SCBA, and Boltzmann theories, the sample size is infinite and the limit $\varepsilon \rightarrow 0$ is taken at the end of the calculation [28-31]. This different order of limits may be responsible for the qualitative difference with Refs. [28-30], which predict a finite conductivity in the limit $\varepsilon \rightarrow 0$. Which order of limits is relevant for experiments depends on the competition between the finite sample size $L$ and the finite temperature or doping $[29,30]$-although the latter is expected to be intrinsically small. The order of the limits $\varepsilon \rightarrow 0$ and $L \rightarrow \infty$ does not affect the comparison to the SCBA, because this theory predicts $\sigma=0$ even if the limit $\varepsilon \rightarrow 0$ is taken at the end of the calculation [31]. Above the critical disorder strength, the self-energy at $\varepsilon=0$ acquires a nonzero (imaginary) value and the order of limits issue is no longer relevant.

Our numerical calculations have shown that the conductance $g$ and the Fano factor $F$ contain important additional information that is not contained in the conductivity $\sigma$. This is particularly relevant for the pseudoballistic weak-disorder regime, where $\sigma$ vanishes, whereas $g$ and $F$ take on nonzero values. A three-dimensional phase with a finite scale-independent bulk conductance is known from the Anderson metal-insulator transition, where it occurs at the critical disorder strength. A crucial difference of the 
pseudoballistic phase at the Weyl point is that its scaleindependent conductance represents an attractive fixed point, which requires no fine-tuning of disorder strength.

We acknowledge helpful discussions with Georg Schwiete, Martin Schneider, Sergey Syzranov, Leo Radzihovsky, Victor Gurarie, and we thank Jörg Behrmann for support with the computations. Financial support was granted by the Helmholtz Virtual Institute "New states of matter and their excitations," by the Alexander von Humboldt Foundation in the framework of the Alexander von Humboldt Professorship, endowed by the Federal Ministry of Education and Research, and by DFG's Emmy Noether program (BE 5233/1-1).

[1] M. Z. Hasan and C. L. Kane, Rev. Mod. Phys. 82, 3045 (2010).

[2] B. A. Bernevig, Topological Insulators and Topological Superconductors (Princeton University Press, Princeton, 2013).

[3] X. G. Wen and A. Zee, Phys. Rev. B 66, 235110 (2002).

[4] P. Hořava, Phys. Rev. Lett. 95, 016405 (2005).

[5] M. Sato, Phys. Rev. B 73, 214502 (2006).

[6] B. Béri, Phys. Rev. B 81, 134515 (2010).

[7] G. Volovik, JETP Lett. 93, 66 (2011).

[8] D. Bernard, E.-A. Kim, and A. LeClair, Phys. Rev. B 86, 205116 (2012).

[9] Y. X. Zhao and Z. D. Wang, Phys. Rev. Lett. 110, 240404 (2013).

[10] S. Matsuura, P.-Y. Chang, A. P. Schnyder, and S. Ryu, New J. Phys. 15, 065001 (2013).

[11] P. R. Wallace, Phys. Rev. 71, 622 (1947).

[12] S. Murakami, New J. Phys. 9, 356 (2007).

[13] X. Wan, A. M. Turner, A. Vishwanath, and S. Y. Savrasov, Phys. Rev. B 83, 205101 (2011).

[14] For a review see P. Hosur and X. Qi, C.R. Phys. 14, 857 (2013).

[15] P. Goswami and S. Tewari, Phys. Rev. B 88, 245107 (2013); M. M. Vazifeh and M. Franz, Phys. Rev. Lett. 111, 027201 (2013).

[16] P. Baireuther, J. M. Edge, I. C. Fulga, C. W. J. Beenakker, and J. Tworzydlo, Phys. Rev. B 89, 035410 (2014).

[17] A. A. Burkov and L. Balents, Phys. Rev. Lett. 107, 127205 (2011).

[18] D. Bulmash, C.-X. Liu, and X.-L. Qi, Phys. Rev. B 89, 081106 (2014).

[19] Y. Xu, R.-L. Chu, and C. Zhang, Phys. Rev. Lett. 112, 136402 (2014).
[20] M. Neupane et al., Nat. Commun. 5, 3786 (2014).

[21] S. Borisenko and Q. Gibson, arXiv:1309.7978.

[22] Z. K. Liu, B. Zhou, Y. Zhang, Z. J. Wang, H. M. Weng, D. Prabhakaran, S.-K. Mo, Z. X. Shen, Z. Fang, X. Dai, Z. Hussain, and Y. L. Chen, Science 343, 864 (2014).

[23] H.-J. Kim, K.-S. Kim, J.-F. Wang, M. Sasaki, N. Satoh, A. Ohnishi, M. Kitaura, M. Yang, and L. Li, Phys. Rev. Lett. 111, 246603 (2013).

[24] The effect of a single impurity on the electronic structure of a Weyl node has been studied recently in Z. Huang, T. Das, A. Balatsky, and D. Arovas, Phys. Rev. B 87, 155123 (2013).

[25] E. Fradkin, Phys. Rev. B 33, 3257 (1986).

[26] E. Fradkin, Phys. Rev. B 33, 3263 (1986).

[27] S. Ryu, A. P. Schnyder, A. Furusaki, and A. W. W. Ludwig, New J. Phys. 12, 065010 (2010).

[28] A. A. Burkov, M. D. Hook, and L. Balents, Phys. Rev. B 84, 235126 (2011)

[29] S. V. Syzranov, L. Radzihovsky, and V. Gurarie, arXiv:1402.3737.

[30] R. R. Biswas and S. Ryu, Phys. Rev. B 89, 014205 (2014).

[31] Y. Ominato and M. Koshino, Phys. Rev. B 89, 054202 (2014).

[32] R. Nandkishore, D. Huse, and S. Sondhi, Phys. Rev. B 89, 245110 (2014).

[33] P. Hosur, S. A. Parameswaran, and A. Vishwanath, Phys. Rev. Lett. 108, 046602 (2012).

[34] K. Kobayashi, T. Ohtsuki, K.-I. Imura, and I. F. Herbut, Phys. Rev. Lett. 112, 016402 (2014).

[35] H. Nielsen and M. Ninomiya, Nucl. Phys. B185, 20 (1981).

[36] J. H. Bardarson, J. Tworzydlo, P. W. Brouwer, and C. W. J. Beenakker, Phys. Rev. Lett. 99, 106801 (2007).

[37] S. Adam, P. W. Brouwer, and S. Das Sarma, Phys. Rev. B 79, 201404 (2009).

[38] J. Tworzydlo, B. Trauzettel, M. Titov, A. Rycerz, and C. W. J. Beenakker, Phys. Rev. Lett. 96, 246802 (2006).

[39] P. Goswami and S. Chakravarty, Phys. Rev. Lett. 107, 196803 (2011).

[40] K. Slevin and T. Ohtsuki, Phys. Rev. Lett. 78, 4083 (1997).

[41] The theory of Ref. [31] can be applied to our calculations by making the substitutions $d_{0} \leftrightarrow \xi$ and $W \leftrightarrow K / 2 \pi$.

[42] J. Rammer, Quantum Transport Theory (Perseus Books, New York, 1998).

[43] See Supplemental Material at http://link.aps.org/ supplemental/10.1103/PhysRevLett.113.026602 for details of this calculation.

[44] S. Das Sarma, E. H. Hwang, and Q. Li, Phys. Rev. B 85, 195451 (2012).

[45] Y. Imry, Introduction to Mesoscopic Physics (Oxford University Press, New York, 2002). 